\title{
Quantitative evaluation of COVID-19 pneumonia severity by CT pneumonia analysis algorithm using deep learning technology and blood test results
}

\author{
Tomohisa Okuma ${ }^{1}$. Shinichi Hamamoto ${ }^{1}$. Tetsunori Maebayashi ${ }^{1}$ - Akishige Taniguchi ${ }^{1} \cdot$ Kyoko Hirakawa $^{1}$. \\ Shu Matsushita ${ }^{1} \cdot$ Kazuki Matsushita $^{1} \cdot$ Katsuko Murata $^{1} \cdot$ Takao Manabe $^{1} \cdot$ Yukio Miki $^{2}$
}

Received: 12 March 2021 / Accepted: 5 May 2021 / Published online: 14 May 2021

(c) Japan Radiological Society 2021

\begin{abstract}
Purpose To evaluate whether early chest computed tomography (CT) lesions quantified by an artificial intelligence (AI)based commercial software and blood test values at the initial presentation can differentiate the severity of COVID-19 pneumonia.

Materials and methods This retrospective study included 100 SARS-CoV-2-positive patients with mild $(n=23)$, moderate $(n=37)$ or severe $(n=40)$ pneumonia classified according to the Japanese guidelines. Univariate Kruskal-Wallis and multivariate ordinal logistic analyses were used to examine whether CT parameters (opacity score, volume of opacity, $\%$ opacity, volume of high opacity, \% high opacity and mean HU total on CT) as well as blood test parameters [procalcitonin, estimated glomerular filtration rate (eGFR), C-reactive protein, \% lymphocyte, ferritin, aspartate aminotransferase, lactate dehydrogenase, alanine aminotransferase, creatine kinase, hemoglobin A1c, prothrombin time, activated partial prothrombin time (APTT), white blood cell count and creatinine] differed by disease severity.

Results All CT parameters and all blood test parameters except procalcitonin and APPT were significantly different among mild, moderate and severe groups. By multivariate analysis, mean HU total and eGFR were two independent factors associated with severity $(p<0.0001)$. Cutoff values for mean HU total and eGFR were, respectively, $-801 \mathrm{HU}$ and $77 \mathrm{ml} / \mathrm{min} / 1.73$ $\mathrm{m}^{2}$ between mild and moderate pneumonia and $-704 \mathrm{HU}$ and $53 \mathrm{ml} / \mathrm{min} / 1.73 \mathrm{~m}^{2}$ between moderate and severe pneumonia. Conclusion The mean HU total of the whole lung, determined by the AI algorithm, and eGFR reflect the severity of COVID19 pneumonia.
\end{abstract}

Keywords COVID-19 · Chest CT · Deep learning · Quantitative analysis

\section{Introduction}

First detected in Wuhan, China in December 2019, the novel coronavirus SARS-CoV-2 infection (COVID-19) has spread rapidly around the world causing a global pandemic [1]. In Japan, more than 400,000 people were infected by February 2021. COVID-19 causes non-specific respiratory symptoms

Tomohisa Okuma

o-kuma@msic.osaka-cu.ac.jp

1 Department of Diagnostic Radiology, Osaka City General Hospital, 2-13-22 Miyakojima-hondori, Miyakojima-ku, Osaka 534-0021, Japan

2 Department of Diagnostic and Interventional Radiology, Graduate School of Medicine, Osaka City University, 1-4-3 Asahi-machi, Abeno-ku, Osaka 545-8585, Japan of varying severity ranging from asymptomatic pneumonia to acute respiratory distress requiring mechanical ventilation. Reported clinical risk factors and predictors for severe illness include age, gender, $\mathrm{C}$-reactive protein (CRP), lactate dehydrogenase (LDH), lymphocyte count, ferritin, d-dimer and comorbidities such as diabetes, chronic obstructive pulmonary disease, hypertension, heart disease, hyperlipidemia and hyperuricemia [2].

The general role of diagnostic imaging is to complement clinical evaluation and laboratory tests in the management of patients already diagnosed with COVID-19 [3]. However, COVID-19 pneumonia can be suspected based on ground-glass opacity (GGO) with or without consolidation on chest computed tomography (CT) even before the results of reverse transcription-polymerase chain reaction (RT-PCR) are available $[4,5]$. The positive 
rate for CT imaging for the diagnosis of suspected COVID-19 patients was $88 \%$ vs. $59 \%$ by RT-PCR, and the sensitivity of CT increased to $97 \%$ based on positive RT-PCR results, confirming the role of $\mathrm{CT}$ as a primary tool for diagnosis [6]. Several studies have shown the ability of visual quantitative evaluation of CT images to predict mortality and severity with high consistency [7-10], and these scoring techniques are now applied to the risk prediction and severity evaluation of COVID-19 pneumonia using artificial intelligence (AI) [11-14]. In these AI-based approaches, the use of routine blood test results is still limited to a few studies. CRP, IL-6, lymphocyte count, neutrophil-to-lymphocyte ratio (NLR) and d-dimer were shown to be correlated with the quantification results of CT images $[15,16]$, while a multivariable regression analysis of clinical and CT parameters showed that the consolidation burden and GGO attenuation were better independent predictors of clinical deterioration and death than CRP and history of heart failure and chronic lung disease [17]. Given the importance of early detection and severity assessment of COVID-19 pneumonia for timely intervention and optimization of outcomes, an AI-based approach combining quantitative $\mathrm{CT}$ image evaluation and blood test results would provide an indispensable tool.

The purpose of the present study was to evaluate whether early CT lesions quantified by an AI-based commercial software and blood test values at the initial presentation can differentiate the severity of COVID-19 pneumonia.

Fig. 1 Flow chart of this study

\section{Materials and methods}

\section{Patients and data}

This retrospective observational study was approved by the institutional review board at our center (No. 2010108). The patient privacy was maintained by anonymization of patient data. Medical records of 107 patients who were suspected of having COVID-19 pneumonia, underwent CT and tested positive for SARS-CoV-2 by RT-PCR at our center between January and November 2020 were included in the study. Seven patients were excluded because of incomplete breathhold during CT, and a total of 100 patients [ 77 men and 23 women; median age, 64 years (interquartile range, IQR, 25)] were included in the further analysis (Fig. 1). The clinical severity was determined according to the guideline of the Ministry of Health, Labour and Welfare of Japan [18] and classified as mild $\left(\mathrm{SpO}_{2}\right.$ of $\geq 96 \%$; coughing only without shortness of breath), moderate $\left(\mathrm{SpO}_{2}\right.$ of $93-96 \%$; shortness of breath with radiologic evidence of pneumonia or requirement for oxygen therapy) and severe (requiring admission to an intensive care unit or artificial ventilation). The highest severity during the disease course was employed for analysis. Blood test parameters that were determined at the time of initial hospital visit, i.e., procalcitonin, estimated glomerular filtration rate (eGFR), CRP, lymphocyte percentage (\% LYM), ferritin, aspartate aminotransferase (AST), LDH, alanine aminotransferase (ALT), creatine kinase (CK), hemoglobin A1c (HbA1c), prothrombin time (PT), activated partial prothrombin time (APPT), white blood cell count (WBC) and creatinine, were included in the analysis.

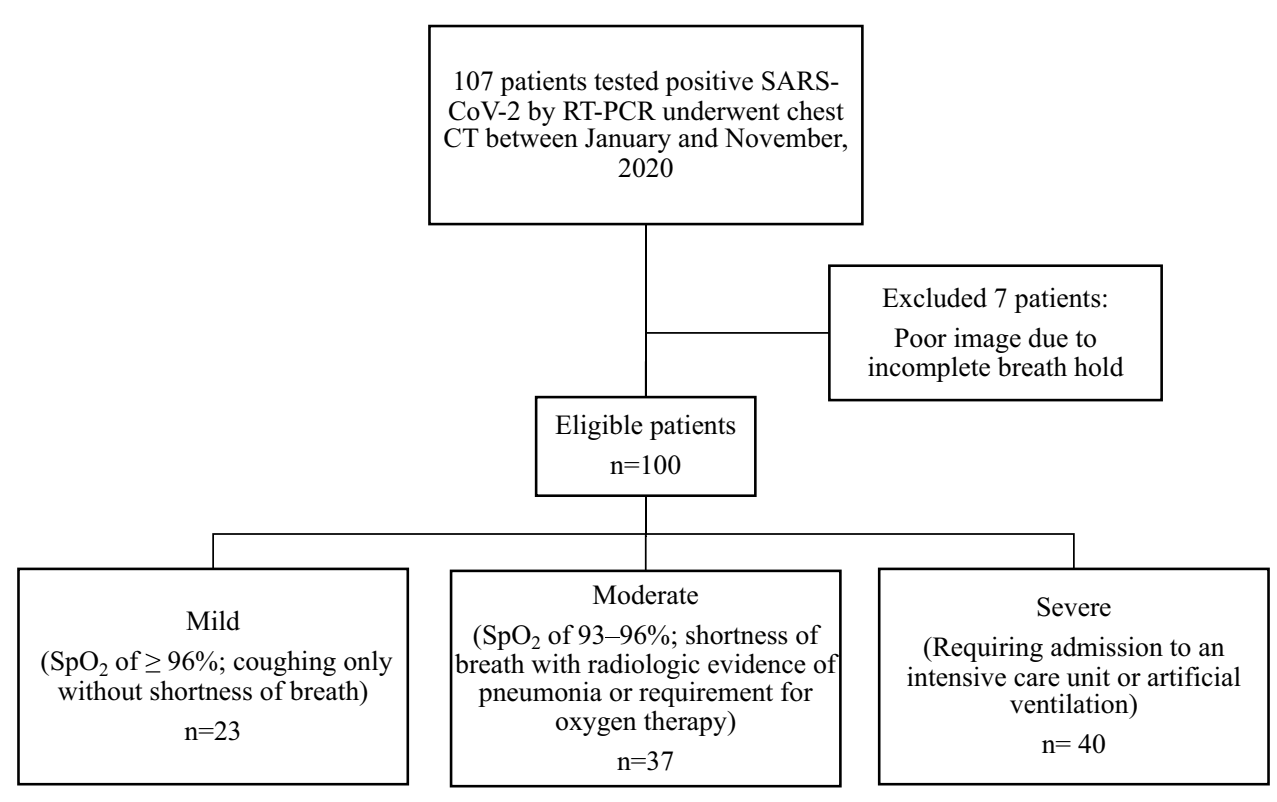




\section{Chest CT protocol}

The first non-contrast CT images taken after the onset of symptoms were included in the study. The median time from PCR diagnosis to CT was 6 days (IQR, 5). Chest CT scans were acquired using a multidetector scanner with 80 detector rows (Aquilion PRIME, Canon Medical Systems, Tochigi, Japan) with the following parameters: $120 \mathrm{kVp}$; automatic tube current modulation; pitch, $0.625 \mathrm{~mm}$; matrix, $512 \times 512$; reconstructed slice thickness, $3 \mathrm{~mm}$; and field of view, $36 \mathrm{~cm}$. CT image data sets were reconstructed with a standard algorithm. The CT dose index volume (CTDI vol; in $\mathrm{mGy}$ ), a standardized measure of the output radiation dose of a CT scanner, was examined.

\section{Al analysis}

CT Pneumonia Analysis (Siemens Healthineers, Erlangen, Germany) was used for the quantitation of chest CT lesions. This AI-based software automatically detects and quantifies GGO and consolidation using the algorithm that has been trained by deep learning. Using non-contrasted chest CT data as input, the algorithm automatically detects GGO and consolidation, performs 3D segmentation of lesions, lungs and lobes and quantifies the extent of overall abnormalities as well as the presence of high opacity abnormalities. The quantitative results included in the present analysis were: the opacity score, which was calculated for each lobe by estimating $\%$ of opacity within a given region $(<1 \%$, score $=0$; $1-25 \%$, score $=1 ; 25-50 \%$, score $=2 ; 50-75 \%$, score $=3$; and $>75 \%$, score $=4$ ), total opacity score (the sum of opacity scores of all lobes), volume of opacity as the absolute value of lung parenchyma affected by infection $(\mathrm{mL}), \%$ of opacity within a given lung region, the volume of high opacity as an absolute value $(\mathrm{mL}), \%$ of high opacity within a given lung region, the mean Hounsfield unit (HU) total of all parenchyma within a given lung region and the mean $\mathrm{HU}$ of opacity within a given lung region. A high opacity region is defined as a region of $\geq-200 \mathrm{HU}$. Figures 2 and 3 show representative $\mathrm{CT}$ features and image by this software.

\section{Statistical analysis}

Data were expressed as median and IQR. Univariate analysis by the Kruskal-Wallis test was performed for comparison of age as well as quantitative chest $\mathrm{CT}$ and blood test variables among three severity groups (mild, moderate and severe). All variables were also checked for multicollinearity, and those with high correlation were excluded from the multivariate analysis that followed. Significant variables based on the univariate analysis were entered into backward stepwise regression analysis to select the 7 most significant variables. The ordinal logistic analysis was used for multivariate analysis since the response variables were ordinal. In addition to stepwise selection, combinations of CT variables (opacity score, $\%$ opacity, $\%$ high opacity, mean HU total and mean HU opacity) and age, eGFR and were analyzed by a multivariate analysis model. Finally, cutoff values for mild, moderate and severe pneumonia were estimated by receiver operating curve (ROC) analysis and Youden's index. All statistical analyses were performed using JMP version 14.2 (SAS Institute, Cary, NC, USA) and PRISM version 8.4 (GraphPad Software, La Jolla, CA, USA). $p$ values less than 0.05 were considered statistically significant.

\section{Results}

Data of 100 patients diagnosed with COVID-19 pneumonia and results of univariate analysis are summarized in Table 1. Pneumonia was mild in 23, moderate in 37 and severe in 40 patients. The median CT dose index volume for patients was $16.2 \mathrm{mGy}(\mathrm{IQR}, 7)$. By the Kruskal-Wallis test, all of the quantitative CT parameters were found to be significant factors $(p<0.05)$. Age as well as eGFR, CRP, \% LYM, ferritin, AST, LDH, CK, HbA1c, PT, WBC and creatinine were also significant $(p<0.05)$. Multiple linear regression analysis showed high correlations between $\%$ opacity and volume of opacity $(\mathrm{r}=0.84)$ and between $\%$ high opacity and volume of high opacity $(r=0.91)$. Volumes of opacity and high opacity were excluded from the multivariate analysis. No other significant correlations were found.

After stepwise regression analysis, \% opacity, mean $\mathrm{HU}$ total, eGFR, WBC, \% LYM, LDH and CK were found to be significant. By multivariate analysis, significant variables were the mean HU total $[p=0.038$; likelihood ratio $\chi^{2}=4.30 ; 95 \%$ confidence interval (CI), -0.0258 to -0.001 ] and eGFR ( $p=0.008$; likelihood ratio $\chi^{2}=7.07 ; 95 \% \mathrm{CI}$, 0.0068 to 0.044 ) (Table 2). Multivariate analysis of combinations also identified the mean HU total $(p=0.0425)$ and eGFR $(p=0.0335)$ as independent factors.

The ROC analysis for all CT quantitative analysis values and blood test results in each binary group are shown in Table 3. For the two factors that were significant in the multivariate analysis, the cutoff values of the mean $\mathrm{HU}$ total were $-801 \mathrm{HU}$ for mild vs. moderate (AUC $=0.75$; Youden's index $=0.47$; sensitivity, 0.82; specificity, $0.65 ; p=0.002$, $95 \%$ confidence interval (CI), 0.61 to 0.88 ) and $-704 \mathrm{HU}$ for moderate vs. severe (AUC $=0.71$; Youden's index $=0.46$; sensitivity, 0.59 ; specificity, $0.86 ; p=0.002,95 \% \mathrm{CI}, 0.59$ to 0.83 ). The cutoff values of eGFR were 77 for mild vs. moderate $(\mathrm{AUC}=0.61$; Youden's index $=0.33$; sensitivity, 0.61 ; specificity, $0.71 ; p=0.16,95 \% \mathrm{CI}, 0.45$ to 0.77$)$ and 53 for moderate vs. severe (AUC $=0.64$; Youden's index $=0.4$; sensitivity, 0.54 ; specificity, $0.86 ; p=0.036,95 \% \mathrm{CI}, 0.51$ to 0.79 ). 
Fig. 2 Chest $\mathrm{CT}$ of a 70 old-male diagnosed severe COVID-19 pneumonia shows consolidation and ground-glass opacity with a crazy-paving appearance in the bilateral lobe. CT pneumonia analysis displays various quantitative values

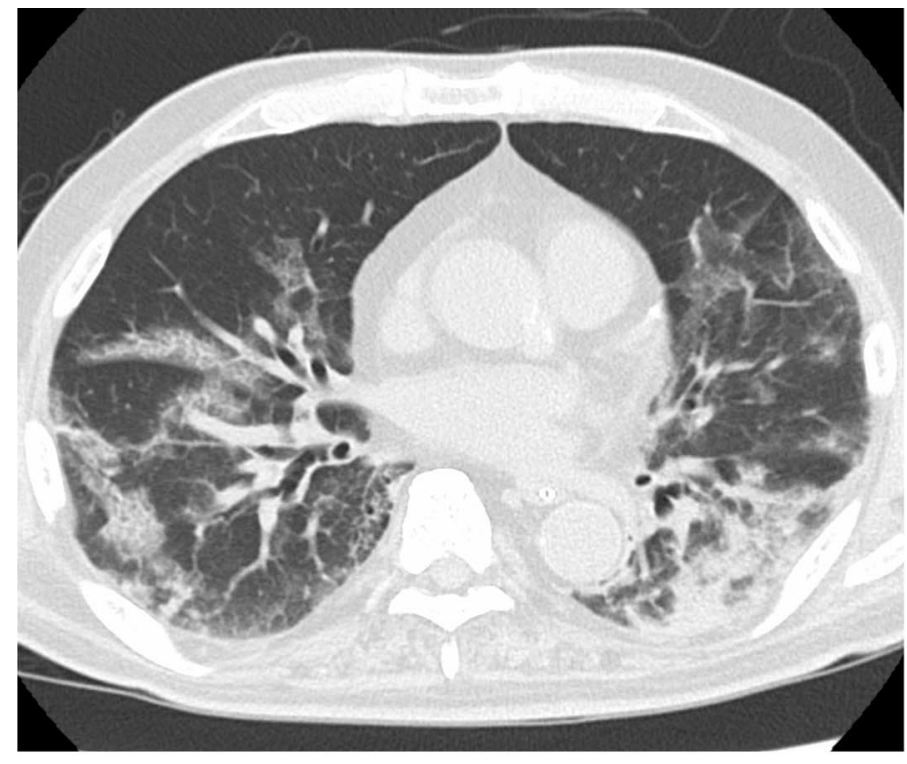

\begin{tabular}{|c|c|c|c|c|c|}
\hline & \multicolumn{2}{|r|}{$\begin{array}{c}\text { Results } \\
\text { Pulmonary Density }\end{array}$} & \multicolumn{2}{|c|}{ Al-Rad Companion } & \\
\hline LUNG OPACITY & Both Lungs & Right Lung & Left Lung & Nat for diannes & \\
\hline Opacity Score & 11 & 5 & 6 & & \\
\hline Total Volume [ml] & 3097.96 & 1778.24 & 1319.72 & & \\
\hline Opacity Volume [ml] & 1444.90 & 704.92 & 739.98 & & \\
\hline Opacity Percentage [\%] & 46.64 & 39.64 & 56.07 & & \\
\hline High Opacity Volume [ml] & 227.64 & 104.02 & 123.62 & & \\
\hline High Opacity Percentage [\%] & 7.35 & 5.85 & 9.37 & & \\
\hline Mean HU Total [HU] & -667.37 & -692.08 & -634.08 & & \\
\hline Mean HU of Opacity [HU] & -521.85 & -525.90 & -517.98 & & \\
\hline Standard Deviation Total [HU] & 282.27 & 277.19 & 285.61 & & \\
\hline Standard Deviation of Opacity [HU] & 271.26 & 268.00 & 274.28 & & \\
\hline LUNG LOBE OPACITY & Right Upper & Right Middle & Right Lower & Left Upper & Left Lower \\
\hline Opacity Score & 2 & 1 & 2 & 3 & 3 \\
\hline Total Volume [ml] & 730.59 & 394.73 & 652.92 & 918.17 & 401.54 \\
\hline Opacity Volume [ml] & 339.31 & 71.60 & 294.01 & 493.27 & 246.70 \\
\hline Opacity Percentage [\%] & 46.44 & 18.14 & 45.03 & 53.72 & 61.44 \\
\hline High Opacity Volume [ml] & 50.10 & 6.01 & 47.91 & 32.39 & 91.23 \\
\hline High Opacity Percentage [\%] & 6.86 & 1.52 & 7.34 & 3.53 & 22.72 \\
\hline Mean HU Total [HU] & -692.14 & -815.90 & -617.14 & -700.01 & -483.32 \\
\hline Mean HU of Opacity [HU] & -533.71 & -607.65 & -496.99 & -600.37 & -353.24 \\
\hline Standard Deviation Total [HU] & 284.98 & 212.86 & 275.88 & 240.56 & 321.12 \\
\hline Standard Deviation of Opacity [HU] & 273.60 & 250.98 & 260.65 & 225.21 & 289.01 \\
\hline
\end{tabular}

\section{Discussion}

By multivariate analysis of chest CT values quantified by a deep learning algorithm and clinical laboratory values, we found that the mean HU total and eGFR are independent factors that reflect the severity of COVID-19 pneumonia $(p<0.0001)$. Early risk identification using these factors is expected to facilitate severity assessment and determine optimal treatment strategies at earlier stages. 
Fig. 3 Chest CT of a 38 oldmale diagnosed with moderate COVID-19 pneumonia shows peripheral ground-glass opacities predominantly in the lower lungs. CT pneumonia analysis displays various quantitative values
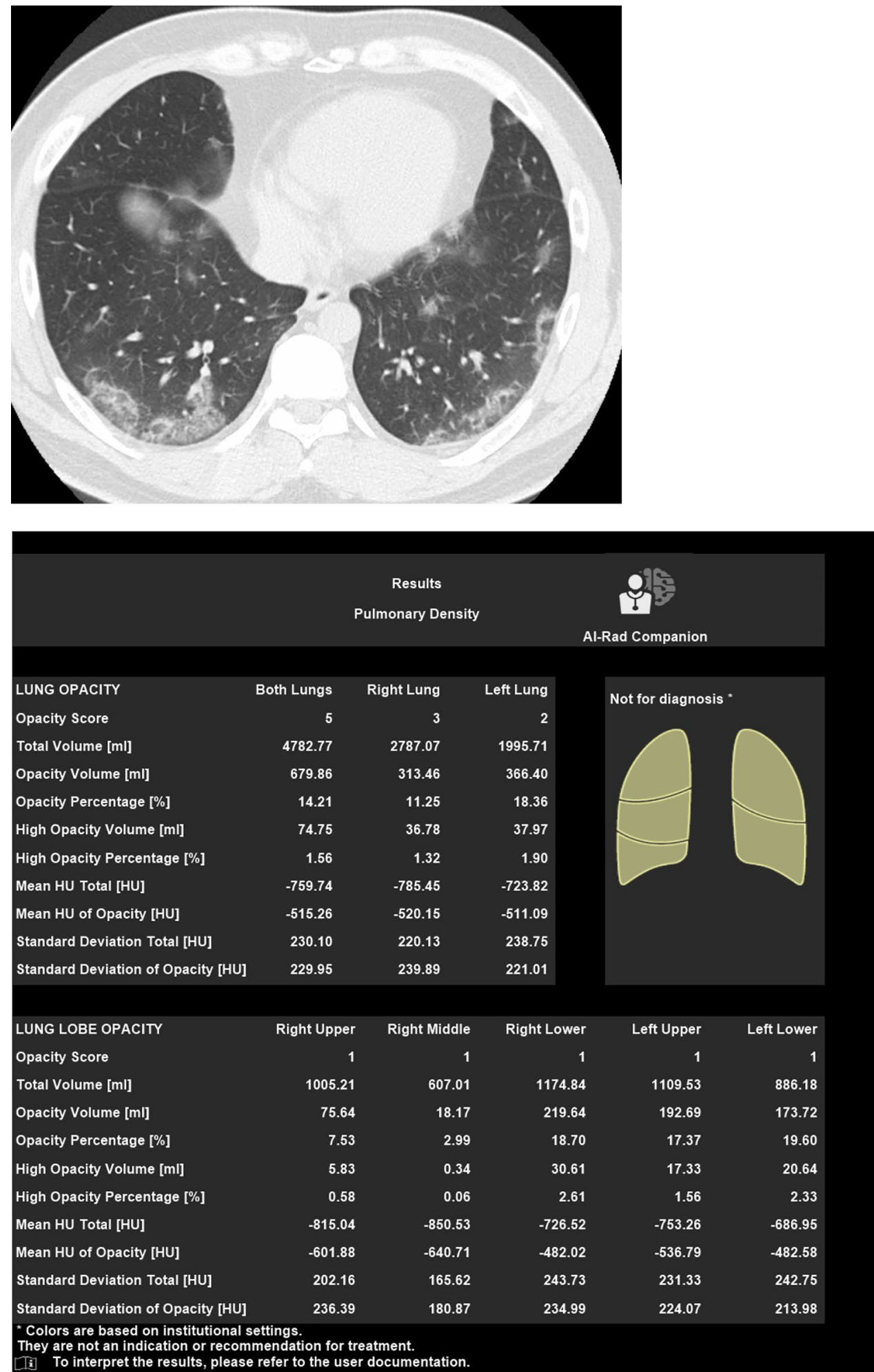

Reported imaging parameters that can predict clinical deterioration include quantitative bilateral consolidation on CT [odds ratio (OR), 4.84] [17] and chest radiographic abnormality (OR, 3.39) [19], and the number of affected lobes is also correlated with prognosis [21]. Yuan et al. reported that the CT score determined by experienced pulmonologists was higher in the mortality group compared to the survival group and concluded that a simple scoring method could predict mortality [7]. Li et al. also reported that visual quantitative evaluation of CT images reflected the 
Table 1 Patient data, CT and blood parameters and $p$ values for group comparisons by Kruskal-Wallis test

\begin{tabular}{|c|c|c|c|c|c|}
\hline & Total $(n=100)$ & Mild (n=23) & Moderate $(n=37)$ & Severe $(n=40)$ & $p$ \\
\hline \multicolumn{6}{|l|}{ Sex } \\
\hline Male & 77 & 13 & 32 & 32 & \\
\hline Female & 23 & 10 & 5 & 8 & \\
\hline Age & $64 \pm 27(22-85)$ & $52 \pm 23(22-77)$ & $65 \pm 27(31-85)$ & $70 \pm 16(29-85)$ & 0.0017 \\
\hline \multicolumn{6}{|l|}{ CT values } \\
\hline Opacity score & $5 \pm 6(0-17)$ & $2 \pm 3(0-12)$ & $5 \pm 6(1-12)$ & $8 \pm 7(0-17)$ & $<0.0001$ \\
\hline Lung volume (ml) & $\begin{array}{l}3738.31 \pm 1438.43 \\
(2268.16-7048.26)\end{array}$ & $\begin{array}{r}4253.33 . \pm 1543.22 \\
(2504.1-6387.95)\end{array}$ & $\begin{array}{l}3858.72 \pm 1351.79 \\
(2541.07-5984.29)\end{array}$ & $\begin{array}{l}3482.93 \pm 886.05 \\
(2268.16-7048.26)\end{array}$ & 0.033 \\
\hline Volume of opacity (ml) & $\begin{array}{l}349.29 \pm 867.25 \\
(0.56-2157.76)\end{array}$ & $\begin{array}{c}153.74 \pm 229.81 \\
(0.56-1743.37)\end{array}$ & $\begin{array}{l}481.83 \pm 835.03 \\
\quad(27.13-1395.03)\end{array}$ & $\begin{array}{r}819.21 \pm 1138.95 \\
(0.98-2157.76)\end{array}$ & $<0.0001$ \\
\hline$\%$ of opacity & $\begin{array}{l}8.21 \pm 25.82(0.011- \\
64.19)\end{array}$ & $4.11 \pm 5.90(0.01-51.58)$ & $\begin{array}{l}10.18 \pm 19.40(0.52- \\
39.84)\end{array}$ & $\begin{array}{l}25.52 \pm 38.01(0.035- \\
64.19)\end{array}$ & $<0.0001$ \\
\hline $\begin{array}{l}\text { Volume of high opacity } \\
\text { (ml) }\end{array}$ & $\begin{array}{l}38.92 \pm 134.29(0.014- \\
471.05)\end{array}$ & $\begin{array}{l}17.50 . \pm 27.01(0.014- \\
303.40)\end{array}$ & $\begin{array}{l}38.45 \pm 80.96(1.29- \\
\quad 420.07)\end{array}$ & $\begin{array}{r}125.61 \pm 227.05 \\
(0.11-471.05)\end{array}$ & $<0.0001$ \\
\hline$\%$ of high opacity & $\begin{array}{l}1.22 \pm 3.83(0.00026- \\
24.24)\end{array}$ & $\begin{array}{l}0.45 \pm 0.689(0.00026- \\
8.71)\end{array}$ & $\begin{array}{l}1.25 \pm 1.61(0.027- \\
14.55)\end{array}$ & $\begin{array}{l}3.78 \pm 6.88(0.0039- \\
24.24)\end{array}$ & $<0.0001$ \\
\hline Mean HU total (HU) & $\begin{array}{r}-770.95 \pm 112.43 \\
(-903.06-630.1)\end{array}$ & $\begin{array}{l}-814.76 \pm 50.99 \\
(-903.06-630.1)\end{array}$ & $\begin{array}{l}-773.25 \pm 51.34 \\
(-845.62-585.5)\end{array}$ & $\begin{array}{l}-692.88 \pm 137.78 \\
(-822.68-501.67)\end{array}$ & $<0.0001$ \\
\hline $\begin{array}{l}\text { Mean HU of opacity } \\
\text { (HU) }\end{array}$ & $\begin{array}{r}-544.11 \pm 129.55 \\
(-751.3-318.09)\end{array}$ & $\begin{array}{r}-588.18 \pm 166.53 \\
(-751.3-361.8)\end{array}$ & $\begin{array}{l}-551.04 \pm 102.76 \\
(-675.99-318.09)\end{array}$ & $\begin{array}{c}-505.35 \pm 151.93 \\
(-664.4-338.69)\end{array}$ & 0.039 \\
\hline \multicolumn{6}{|l|}{ Blood test values } \\
\hline Procalcitonin (ng/ml) & $0.09 \pm 0.10(0.02-29)$ & $0.06 \pm 0.085(0.02-29)$ & $0.08 \pm 0.07(0.02-0.43)$ & $0.13 \pm 0.16(0.03-2.3)$ & 0.28 \\
\hline $\mathrm{eGFR}\left(\mathrm{ml} / \mathrm{min} / 1.73 \mathrm{~m}^{2}\right)$ & $69.5 \pm 38.25(6-145)$ & $78.15 \pm 21.58(31-145)$ & $70.28 \pm 22.3(6-120)$ & $50.00 . \pm 44.75(8 .-110)$ & 0.008 \\
\hline $\begin{array}{l}\text { C-reactive protein (mg/ } \\
\text { dl) }\end{array}$ & $4.89 \pm 7.22(0.07-24.25)$ & $1.79 \pm 3.15(0.07-10.83)$ & $5.21 \pm 6.11(0.31-24.25)$ & $6.76 \pm 6.80(0.31-22.15)$ & $<0.0001$ \\
\hline \% Lymphocyte & $18.15 \pm 16.48(0.8-54.7)$ & $23.05 \pm 12.63(9-54.7)$ & $16.7 \pm 17.15(0.8-36)$ & $14.9 \pm 14.25(4-53.6)$ & 0.0075 \\
\hline Ferritin (ng/ml) & $827.5 \pm 895.75(4-6667)$ & $546 \pm 523.25(4-886)$ & $1003 \pm 1073(31-6667)$ & $868.66 \pm 834(222-6000)$ & 0.0041 \\
\hline AST (IU/l) & $38 \pm 29.75(14-151)$ & $27 \pm 21.75(14-121)$ & $38 \pm 23(22-79)$ & $43 \pm 40.5(16-151)$ & 0.0038 \\
\hline LDH (U/l) & $327.83 \pm 184(75-1173)$ & $\begin{array}{l}263.23 \pm 100.25 \\
(75-1173)\end{array}$ & $309.95 \pm 150(144-535)$ & $\begin{array}{l}392.86 \pm 243.25 \\
(80-1112)\end{array}$ & 0.0006 \\
\hline ALT (IU/l) & $30 \pm 22.5(10-391)$ & $24.5 \pm 23(10-258)$ & $33 \pm 23(10-391)$ & $31 \pm 21.75(12-168)$ & 0.39 \\
\hline CK (U/l) & $85 \pm 126(0.79-3008)$ & $62 \pm 38(28-583)$ & $\begin{array}{l}74.5 \pm 139.25(0.79- \\
2133)\end{array}$ & $126 \pm 238(29-3008)$ & 0.0022 \\
\hline HbA1c (\%) & $6.1 \pm 1.3(5-13.4)$ & $5.85 \pm 1.3(5-11.4)$ & $5.9 \pm 1.1(5-10.8)$ & $6.4 \pm 1.08(5.4-13.4)$ & 0.013 \\
\hline PT (sec) & $11 \pm 1.05(9.5-99.3)$ & $10.9 \pm 0.45(9.6-12.3)$ & $10.8 \pm 1.15(9.5-99.3)$ & $11.4 \pm 1.85(10.2-95)$ & 0.022 \\
\hline APTT (sec) & $33.1 \pm 7(24.4-73.8)$ & $33.1 \pm 5.15(25.2-46.9)$ & $32.55 \pm 6.95(27.4-73.8)$ & $35.25 \pm 8.08(24.4-64.1)$ & 0.36 \\
\hline WBC $\left(\times 10^{9} / 1\right)$ & $5.6 \pm 3.05(1.88-33.3)$ & $4.3 \pm 2.59(2.81-33.3)$ & $5.26 \pm .2 .61(1.88-13.65)$ & $6.35 \pm 3.66(2.65-12.59)$ & 0.007 \\
\hline Creatinine (mg/dl) & $0.86 \pm 0.45(0.43-5396)$ & $0.76 \pm 0.34(0.52-1.91)$ & $0.85 \pm 0.18(0.43-5396)$ & $1.07 \pm 0.63(0.55-5.91)$ & 0.013 \\
\hline
\end{tabular}

Data are expressed as median \pm IQR (range), and $p$ values less than 0.05 were considered significant

$e G F R$ estimated glomerular filtration rate, $A S T$ aspartate aminotransferase, $L D H$ lactate dehydrogenase, $A L T$ alanine aminotransferase, $C K$ creatine kinase, $H b A l c$ hemoglobin A1c, $P T$ prothrombin time, $A P T T$ activated partial prothrombin time, $W B C$ white blood cell count

severity of COVID-19 [8]. In a study by Zhou et al., the total CT score was one of the independent risk factors for poor prognosis, and temporal changes of CT findings and severity scores were important factors for the early identification of severe cases, which would help to minimize the mortality rate [9]. We did not perform a qualitative, visual assessment of CT images and instead used an AI-based quantitative analysis which is increasingly employed for accurate severity stratification of COVID-19 patients. In a study by
Mergen et al. [15], \% opacity and \% high opacity were significantly higher in patients requiring In a study by Mergen et al. [15], \% opacity and \% high opacity were significantly higher in patients requiring mechanical ventilation and moderately correlated with CRP $(\mathrm{r}=0.49-0.60$, both $p<0.001)$ and WBC $(\mathrm{r}=0.30-0.40$, both $p=0.05)$. The $\%$ opacity was also negatively correlated with $\mathrm{SpO}_{2}$. In a study using the same CT Pneumonia Analysis software, the opacity score, $\%$ opacity, volume of opacity, volume of high opacity, $\%$ high 
Table 2 Chi-square and $p$ values and $95 \%$ confidence interval (CI) for multivariate analysis

\begin{tabular}{llllr}
\hline & Chi-square & $p$ & Lower 95\% CI & Upper 95\% CI \\
\hline Percentage of opacity & 0.00 & 0.980 & -0.057 & 0.054 \\
Mean HU total* & 4.30 & 0.038 & -0.026 & -0.001 \\
eGFR* & 7.07 & 0.008 & 0.0068 & 0.044 \\
White blood cell count & 1.38 & 0.240 & -0.061 & 0.206 \\
\% Lymphocyte & 1.99 & 0.159 & -0.012 & 0.074 \\
LDH & 1.38 & 0.239 & -0.0053 & 0.001 \\
CK & 2.68 & 0.102 & -0.007 & 0.000
\end{tabular}

eGFR estimated glomerular filtration rate, $W B C$ white blood cell count, $L D H$ lactate dehydrogenase, $C K$ creatine kinase, $H b A l c$ hemoglobin A1c

$* p>0.05$ opacity and mean HU total were significantly higher in the moderate and severe groups compared to the mild group, while the total lung volume was significantly lower in the severe group compared to the mild group [14]. The results of our study also showed significant differences in \% opacity and $\%$ of high opacity in univariate analysis, which is consistent with their results. In our study, however, the mean HU of opacity was not significantly different between moderate and severe patients. This is probably because different clinical criteria were used for moderate and severe classification. Nonetheless, results in their study and ours suggest that AIbased quantitative CT assessment is a valuable early tool with high sensitivity and specificity for severity assessment. It is expected to play an important role in early COVID-19 management.

The results of the multivariate analysis in our study showed that a higher mean HU total, i.e., higher density in both lungs, is more likely to be associated with severe disease. GGO in the dorsal aspect of the lower lobe accompanied by reduced lung volume is a characteristic CT finding in COVID-19 pneumonia, and consolidation is more frequently found in severer cases [22]. In one study, which also used AI for the quantification of CT findings, multivariate analysis showed that \% GGO, semi-consolidation volume and consolidation volume were significantly better predictors of severity than NLR or d-dimer [16]. Another AI-based CT study evaluated the CT severity score, GGO volume, \% GGO volume, consolidation volume and \% consolidation volume and found that the consolidation volume was a strong predictor for unfavorable outcome by multivariate regression analysis (hazard ratio, 1.053; $p=0.006$ ) [13]. Although different parameters are available in the AI analysis software we used, the mean HU total was associated with severity. Collectively, these findings strongly indicate the usefulness of quantitative CT lesion assessment in COVID-19 pneumonia.

Reported cutoff values for the mean HU total to differentiate mild from moderate and severe cases were -637.7 (AUC, $0.876 ; 81.8 \%$ sensitivity and $81.9 \%$ specificity) [14] and -816 (AUC, $0.87 ; 91 \%$ sensitivity and $90 \%$ specificity)
[23]. In these studies, mild pneumonia included patients with respiratory symptoms and evidence of pneumonia on $\mathrm{CT}$. In our study, the cutoff value was - 801 HU for differentiation of mild $\left(\mathrm{SpO}_{2}\right.$ of $\geq 96 \%$; coughing only with no shortness of breath) from moderate pneumonia $\left(\mathrm{SpO}_{2}\right.$ of $93-96 \%$; shortness of breath with radiographic evidence of pneumonia or requirement for oxygen therapy). These results indicate that cutoff values for the mean HU total are likely able to differentiate mild, moderate and severe diseases early in the disease course. The ability to predict clinical deterioration based on the earliest CT images should assist early identification of and early intervention for patients who are likely to develop severer pneumonia.

Factors associated with severer COVID-19 have been extensively studied and reviewed. These include age, comorbidities such as chronic obstructive pulmonary disease, diabetes, hypertension, chronic kidney disease and malignancy, sequential organ failure assessment score and history of cancer. Among blood test parameters, NLR, LDH, direct bilirubin, albumin, creatinine, d-dimer, LDH, AST, ALT, BUN, creatinine, procalcitonin, IL-6, KL-6 and ferritin have been associated with severity [24-26]. Although we did not include comorbidities in our analysis, we found that eGFR, CRP, \% LYM, ferritin, AST, LDH, CK, HbA1c and PT were significantly associated with disease severity. By multivariate analysis, we found that eGFR was the most significant factor among them. One study reported that a high percentage of COVID-19 patients had renal abnormalities and that although the majority of proteinuria, hematuria and acute kidney injury resolved within 3 weeks of the onset of symptoms, renal involvement was associated with higher mortality [27]. Thus, renal function impairment in the early stage of COVID-19 is likely associated with the disease severity. The role of early severity assessment based on initial blood test results s and CT values quantified by a deep learning algorithm are significant, as physicians would be better prepared for early intervention before clinical deterioration occurs and able to provide appropriate treatment at the time of deterioration with watchful waiting. If the usefulness of 
Table 3 Cutoff values for CT and blood test to differentiate mild from moderate and severe cases

\begin{tabular}{|c|c|c|c|c|c|c|c|c|}
\hline Mild vs moderate & Cut off & AUC & Sensitivity & Specificity & Youden's index & Lower 95\% CI & Upper 95\% CI & $p$ \\
\hline \multicolumn{9}{|l|}{ CT values } \\
\hline Opacity Score* & 3.00 & 0.76 & 0.84 & 0.54 & 0.38 & 0.64 & 0.88 & 0.000 \\
\hline Volume of opacity* & 473.3 & 0.73 & 0.54 & 0.92 & 0.46 & 0.60 & 0.86 & 0.002 \\
\hline Percentage of opacity* & 9.48 & 0.72 & 0.54 & 0.88 & 0.43 & 0.59 & 0.85 & 0.003 \\
\hline Volume of high opacity* & 32.25 & 0.70 & 0.59 & 0.77 & 0.36 & 0.57 & 0.84 & 0.006 \\
\hline Percentage of high opacity* & 0.94 & 0.69 & 0.57 & 0.77 & 0.34 & 0.56 & 0.83 & 0.010 \\
\hline Mean HU total* & -801 & 0.75 & 0.82 & 0.65 & 0.46 & 0.62 & 0.88 & 0.002 \\
\hline Mean HU of opacity & -612 & 0.61 & 0.84 & 0.46 & 0.24 & 0.46 & 0.76 & 0.150 \\
\hline \multicolumn{9}{|l|}{ Blood test values } \\
\hline Procalcitonin & 0.43 & 0.42 & 1.00 & 0.18 & 0.18 & 0.39 & 0.77 & 0.367 \\
\hline eGFR & 77 & 0.61 & 0.61 & 0.71 & 0.33 & 0.46 & 0.77 & 0.164 \\
\hline C-reactive protein $*$ & 3.73 & 0.80 & 0.78 & 0.69 & 0.53 & 0.69 & 0.91 & $<0.0001$ \\
\hline Percentage of Lymphocyte* & 13.50 & 0.67 & 0.47 & 0.88 & 0.36 & 0.54 & 0.81 & 0.020 \\
\hline Ferritin* & 932 & 0.75 & 0.55 & 1.00 & 0.55 & 0.61 & 0.88 & 0.004 \\
\hline $\mathrm{AST}^{*}$ & 29 & 0.68 & 0.81 & 0.54 & 0.35 & 0.55 & 0.83 & 0.011 \\
\hline $\mathrm{LDH}^{*}$ & 301 & 0.71 & 0.54 & 0.85 & 0.39 & 0.44 & 0.74 & 0.004 \\
\hline ALT & 28 & 0.59 & 0.65 & 0.62 & 0.26 & 0.44 & 0.74 & 0.238 \\
\hline $\mathrm{CK}$ & 170 & 0.61 & 0.30 & 0.96 & 0.26 & 0.47 & 0.75 & 0.151 \\
\hline $\mathrm{HbA1c}$ & 9.8 & 0.48 & 0.97 & 0.17 & 0.14 & 0.34 & 0.69 & 0.848 \\
\hline PT & 11.30 & 0.48 & 0.34 & 0.84 & 0.19 & 0.35 & 0.68 & 0.856 \\
\hline APTT & 27.40 & 0.50 & 1.00 & 0.16 & 0.16 & 0.34 & 0.67 & 0.979 \\
\hline WBC count & 2.62 & 0.43 & 0.08 & 1.00 & 0.08 & 0.43 & 0.72 & 0.335 \\
\hline Creatinine & 0.79 & 0.60 & 0.75 & 0.58 & 0.33 & 0.44 & 0.75 & 0.192 \\
\hline Moderate vs severe & Cut off & AUC & Sensitivity & Specificity & Youden's index & Lower 95\% CI & Upper $95 \%$ CI & $p$ value \\
\hline \multicolumn{9}{|l|}{ CT values } \\
\hline Opacity Score* & 11 & 0.67 & 0.38 & 0.95 & 0.32 & 0.55 & 0.80 & 0.011 \\
\hline Volume of opacity & 1405.55 & 0.64 & 0.32 & 100.00 & 0.32 & 0.49 & 0.75 & 0.078 \\
\hline Percentage of opacity* & 37.84 & 0.68 & 0.43 & 0.95 & 0.38 & 0.56 & 0.80 & 0.008 \\
\hline Volume of high opacity* & 101.05 & 0.66 & 0.62 & 0.81 & 0.43 & 0.53 & 0.79 & 0.020 \\
\hline Percentage of high opacity* & 2.11 & 0.68 & 0.65 & 0.78 & 0.43 & 0.55 & 0.81 & 0.008 \\
\hline Mean HU total* & -704 & 0.71 & 0.59 & 0.86 & 0.46 & 0.59 & 0.83 & 0.002 \\
\hline Mean HU of opacity & -482 & 0.60 & 0.43 & 0.76 & 0.24 & 0.47 & 0.73 & 0.134 \\
\hline \multicolumn{9}{|l|}{ Blood test values } \\
\hline Procalcitonin* & 95 & 0.33 & 0.97 & 0.03 & 0.03 & 0.57 & 0.82 & 0.047 \\
\hline $\mathrm{eGFR}^{*}$ & 53 & 0.64 & 0.85 & 0.54 & 0.40 & 0.51 & 0.79 & 0.036 \\
\hline C-reactive protein & 6.67 & 0.65 & 0.67 & 0.57 & 0.26 & 0.50 & 0.76 & 0.064 \\
\hline Percentage of Lymphocyte & 25.7 & 0.54 & 0.86 & 0.28 & 0.14 & 0.39 & 0.66 & 0.706 \\
\hline Ferritin & 293 & 0.50 & 0.97 & 0.15 & 0.12 & 0.36 & 0.64 & 0.980 \\
\hline AST & 61 & 0.61 & 0.43 & 0.84 & 0.27 & 0.49 & 0.76 & 0.064 \\
\hline $\mathrm{LDH}^{*}$ & 422 & 0.62 & 0.44 & 0.77 & 0.28 & 0.52 & 0.77 & 0.031 \\
\hline ALT & 34 & 0.51 & 0.64 & 0.46 & 0.10 & 0.38 & 0.65 & 0.817 \\
\hline $\mathrm{CK}^{*}$ & 113 & 0.64 & 0.64 & 0.68 & 0.31 & 0.54 & 0.79 & 0.015 \\
\hline HbA1c* & 6.1 & 0.69 & 0.77 & 0.65 & 0.38 & 0.54 & 0.81 & 0.017 \\
\hline $\mathrm{PT}^{*}$ & 0.13 & 0.66 & 0.52 & 0.79 & 0.30 & 0.51 & 0.80 & 0.005 \\
\hline APTT* & 37.3 & 0.61 & 0.42 & 0.75 & 0.23 & 0.51 & 0.77 & 0.048 \\
\hline WBC count & 5.6 & 0.65 & 0.69 & 0.59 & 0.29 & 0.50 & 0.76 & 0.058 \\
\hline Creatinine* & 1.13 & 0.65 & 0.51 & 0.85 & 0.37 & 0.52 & 0.79 & 0.028 \\
\hline
\end{tabular}

$A U C$ area under the curve, $e G F R$ estimated glomerular filtration rate, $A S T$ aspartate aminotransferase, $L D H$ lactate dehydrogenase, $A L T$ alanine aminotransferase, $C K$ creatine kinase, $H b A l c$ hemoglobin A1c, $P T$ prothrombin time, APTT activated partial prothrombin time, WBC white blood cell count

${ }^{*} p>0.05$ 
these results in clinical practice is demonstrated by further studies, it will allow us to evaluate the severity of COVID19 patients, determine whether they should be hospitalized or stay at home and allocate available medical resources.

The present study has some limitations. First, it is a single-center study involving a relatively small number of patients. Second, our center primarily accepts severe COVID-19 patients, and therefore the study results may be biased toward severe cases. Third, the patients in this study were classified according to the greatest severity they experienced during the course of the disease, while only the earliest CT data were used for AI analysis. CT data later in the disease course were not included. In some patients, the condition may deteriorate quite rapidly with acutely progressive GGO, even though GGO was minimal on initial CT assessment. Fourth, the small number of samples did not allow simultaneous multivariate analysis of all variables. Although stepwise regression has both advantages and disadvantages, multivariate analysis of combinations of CT data and inflammatory markers also demonstrated that the mean HU total and eGFR were the independent predictors. Therefore, it was considered a meaningful approach as part of the sensitivity analysis even with a limited number of cases.

\section{Conclusion}

We found that the mean density of the whole lung, as determined by the deep learning algorithm, and eGFR were significant predictors of the severity of COVID-19 pneumonia.

\section{Declarations}

Conflict of interest The authors declare that they have no conflict of interest.

Ethical approval This work was approved by the local institutional review board. No. 2010108.

\section{References}

1. Chen N, Zhou M, Dong X, Qu J, Gong F, Han Y, et al. Epidemiological and clinical characteristics of 99 cases of 2019 novel coronavirus pneumonia in Wuhan, China: a descriptive study. Lancet. 2020;395:507-13.

2. Wynants L, Van Calster B, Collins GS, Riley RD, Heinze G, Schuit E, et al. Prediction models for diagnosis and prognosis of COVID-19 infection: systematic review and critical appraisal. BMJ. 2020; 369:m1328. https://doi.org/10.1136/bmj.m1328.

3. World Health Organization. Clinical management of COVID-19. Interim guidance. 27 May 2020. https://www.who.int/publicatio ns-detail/clinical-management-of-covid-19. Accessed 28 May 2020 .
4. Himoto Y, Sakata A, Kirita M, Hiroi T, Kobayashi K, Kubo $\mathrm{K}$, et al. Diagnostic performance of chest CT to differentiate COVID-19 pneumonia in non-high-epidemic area in Japan. Jpn J Radiol. 2020;38:400-6.

5. Cellina M, Orsi M, Valenti Pittino C, Toluian T, Oliva G. Chest computed tomography findings of COVID-19 pneumonia: pictorial essay with literature review. Jpn J Radiol. 2020;38:1012-9.

6. Ai T, Yang Z, Hou H, Zhan C, Chen C, Lv W, et al. Correlation of chest CT and RT-PCR testing for coronavirus disease 2019 (COVID-19) in China: a report of 1014 cases. Radiology. 2020;296:E32-40.

7. Yuan M, Yin W, Tao Z, Tan W, Hu Y. Association of radiologic findings with mortality of patients infected with 2019 novel coronavirus in Wuhan, China. PLoS ONE. 2020;15:e0230548.

8. Li K, Fang Y, Li W, Pan C, Qin P, Zhong Y, et al. CT image visual quantitative evaluation and clinical classification of coronavirus disease (COVID-19). Eur Radiol. 2020;30:4407-16.

9. Zhou S, Chen C, Hu Y, Lv W, Ai T, Xia L. Chest CT imaging features and severity scores as biomarkers for prognostic prediction in patients with COVID-19. Ann Transl Med. 2020;8:1449.

10. Xiong Y, Sun D, Liu Y, Fan Y, Zhao L, Li X, et al. Clinical and high-resolution CT features of the COVID-19 infection: comparison of the initial and follow-up changes. Invest Radiol. 2020;55:332-9.

11. Li Z, Zhong Z, Li Y, Zhang T, Gao L, Jin D, et al. From community-acquired pneumonia to COVID-19: a deep learning-based method for quantitative analysis of COVID-19 on thick-section CT scans. Eur Radiol. 2020;30:6828-37.

12. Zhang Y, Liu Y, Gong H, Wu L. Quantitative lung lesion features and temporal changes on chest $\mathrm{CT}$ in patients with common and severe SARS-CoV-2 pneumonia. PLoS ONE. 2020;15:e0236858.

13. Li Y, Shang K, Bian W, He L, Fan Y, Ren T, et al. Prediction of disease progression in patients with COVID-19 by artificial intelligence assisted lesion quantification. Sci Rep. 2020;10:22083.

14. Gouda W, Yasin R. COVID-19 disease: CT Pneumonia Analysis prototype by using artificial intelligence, predicting the disease severity. Egypt J Radiol Nucl Med. 2020;51:196.

15. Mergen V, Kobe A, Blüthgen C, Euler A, Flohr T, Frauenfelder $\mathrm{T}$, et al. Deep learning for automatic quantification of lung abnormalities in COVID-19 patients: first experience and correlation with clinical parameters. Eur J Radiol Open. 2020;7:100272

16. Liu F, Zhang Q, Huang C, Shi C, Wang L, Shi N, et al. CT quantification of pneumonia lesions in early days predicts progression to severe illness in a cohort of COVID-19 patients. Theranostics. 2020;10:5613-22.

17. Grodecki K, Lin A, Cadet S, McElhinney PA, Razipour A, Chan C, et al. Quantitative burden of COVID-19 pneumonia on chest CT predicts adverse outcomes: a post-hoc analysis of a prospective international registry. Radiol Cardiothorac Imaging. 2020. https://doi.org/10.1148/ryct.2020200389.

18. Ministry of Health, Labour and Welfare of Japan. Practice guideline for novel coronavirus infection (COVID-19). Version 4.1. 25 December 2020. https://www.mhlw.go.jp/content/000712473.pdf. Accessed $18 \mathrm{Feb} 2021$.

19. Liang W, Liang H, Ou L, Chen B, Chen A, Li C, et al. Development and validation of a clinical risk score to predict the occurrence of critical illness in hospitalized patients with COVID-19. JAMA Intern Med. 2020;180:1081-9.

20. Zhang JJ, Cao YY, Tan G, Dong X, Wang BC, Lin J, et al. Clinical, radiological, and laboratory characteristics and risk factors for severity and mortality of 289 hospitalized COVID-19 patients. Allergy. 2021;76:533-50.

21. Shi H, Han X, Jiang N, Cao Y, Alwalid O, Gu J, et al. Radiological findings from 81 patients with COVID-19 pneumonia in Wuhan, China: a descriptive study. Lancet Infect Dis. 2020;20:425-34. 
22. Zhang T, Huang WS, Guan W, Hong Z, Gao J, Gao G, et al. Risk factors and predictors associated with the severity of COVID-19 in China: a systematic review, meta-analysis, and meta-regression. J Thorac Dis. 2020;12:7429-41.

23. Lyu P, Liu X, Zhang R, Shi L, Gao J. The performance of chest CT in evaluating the clinical severity of COVID-19 pneumonia: identifying critical cases based on CT characteristics. Invest Radiol. 2020;55:412-42.

24. Guan WJ, Liang WH, Zhao Y, Liang HR, Chen ZS, Li YM, et al. Comorbidity and its impact on 1590 patients with COVID-19 in China: a nationwide analysis. Eur Respir J. 2020;55:2000547.

25. Gao YD, Ding M, Dong X, Zhang JJ, Kursat Azkur A, Azkur D, et al. Risk factors for severe and critically ill COVID-19 patients: a review. Allergy. 2020;76:428-55.
26. Zheng Z, Peng F, Xu B, Zhao J, Liu H, Peng J, et al. Risk factors of critical \& mortal COVID-19 cases: a systematic literature review and meta-analysis. J Infect. 2020;81:e16-25.

27. Pei G, Zhang Z, Peng J, Liu L, Zhang C, Yu C, et al. Renal involvement and early prognosis in patients with COVID-19 pneumonia. J Am Soc Nephrol. 2020;31:1157-65.

Publisher's Note Springer Nature remains neutral with regard to jurisdictional claims in published maps and institutional affiliations. 